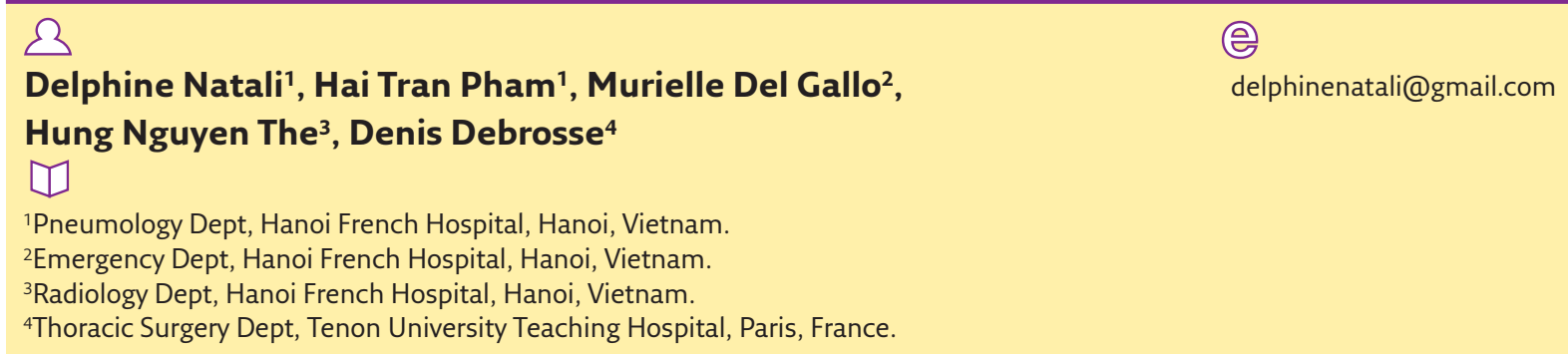

\title{
An unusual pneumonia
}

\section{Case report}

Cite as: Natali D, Tran Pham H, Del Gallo M, et al. An unusual pneumonia. Breathe 2018; 14: $225-228$.
A 70-year-old patient presented to the emergency department with a productive cough and fever for 15 days. He had no general status alteration, no chest pain, no dyspnoea, no haemoptysis and no other symptoms. He was a current cigar smoker, with a >20 pack-years smoking history, and had a past medical history of high blood pressure treated with telmisartan and a polypectomy for benign colon polyp 1 year ago. He had no professional exposures. He was French and had lived in Hanoi, Vietnam, for $>10$ years.

Physical examination showed decreasing transcutaneous arterial saturation to $91 \%$ in room air, normal cardiac frequency and blood pressure, fever up to $38.5^{\circ} \mathrm{C}$, and a diminished vesicular murmur with dullness at left base of the lung.

Blood tests showed important inflammation status with white blood cells $20.4 \times 10^{3}$ cells $\mu \mathrm{L}^{-1}$, neutrophils $16.9 \times 10^{3}$ cells $\mu \mathrm{L}^{-1}$, lymphocytes $1.7 \times 10^{3}$ cells $\mu \mathrm{L}^{-1}$ and C-reactive protein $276 \mathrm{mg} \cdot \mathrm{L}^{-1}$. Renal and liver functions were normal.
Arterial blood gases showed an arterial oxygen tension of $70 \mathrm{mmHg}$, arterial carbon dioxide tension of $37 \mathrm{mmHg}, \mathrm{pH} 7.48$ and $\mathrm{HCO}_{3}{ }^{-} 37 \mathrm{mmol} \cdot \mathrm{L}^{-1}$.

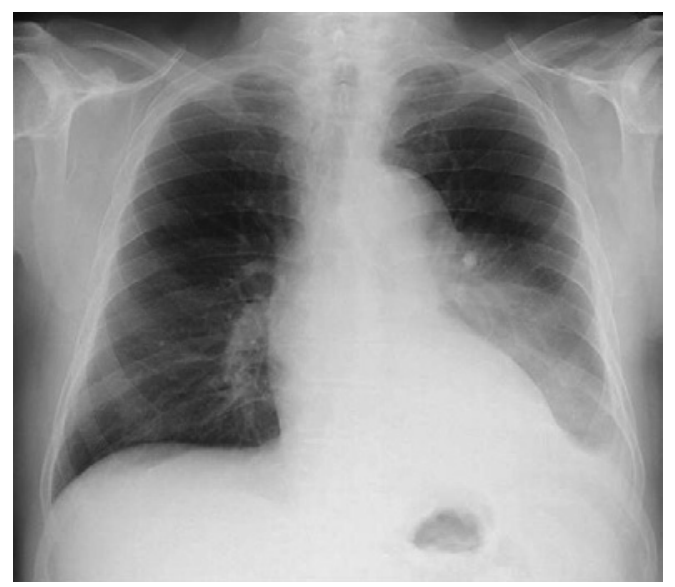

Figure 1 Chest radiograph.

Task 1

Describe the imaging findings on the chest radiograph (figure 1 ). What is your diagnosis? 


\section{Answer 1}

The chest radiograph (figure 2 ) shows a left non-systematised opacity (circled) and a small left pleural effusion (arrow).

Fever, cough, left lower lobe dullness and diminution of the right vesicular murmur, and increased white blood cells and C-reactive protein are consistent with the diagnosis of acute left pneumonia with reactional left pleural effusion. However, the subacute presentation of 15 days is atypical, and the left opacity is not well systematised on the chest radiograph. In a patient that is a current smoker an obstructing process in the proximal bronchial tree should be suspected.
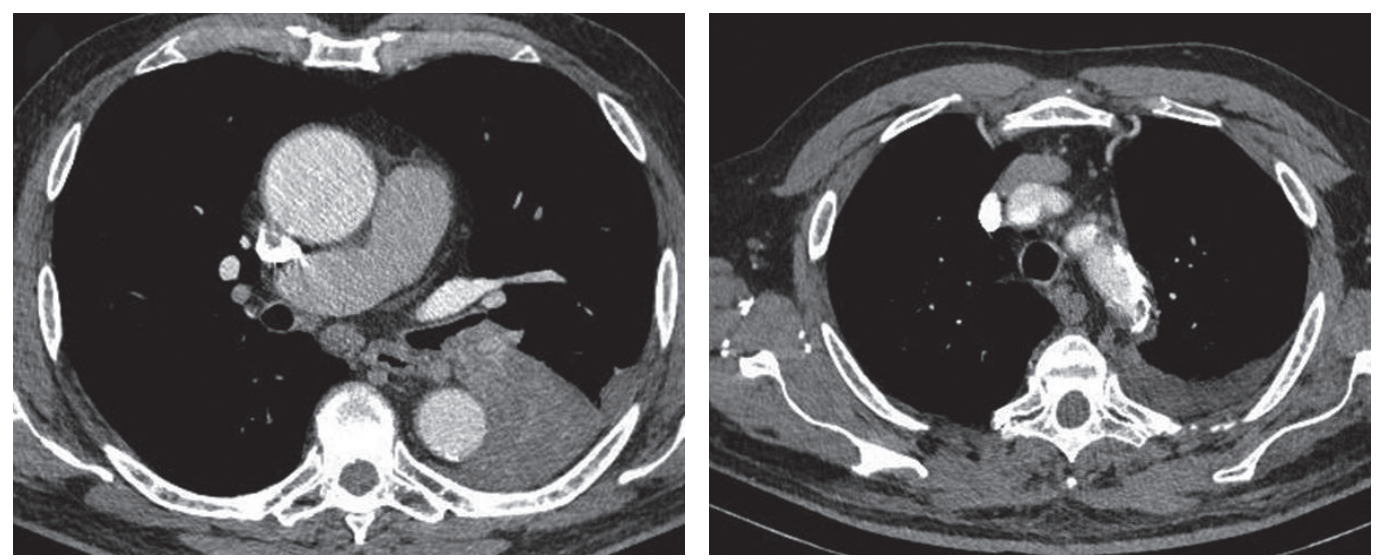

Figure 3 CT images. 

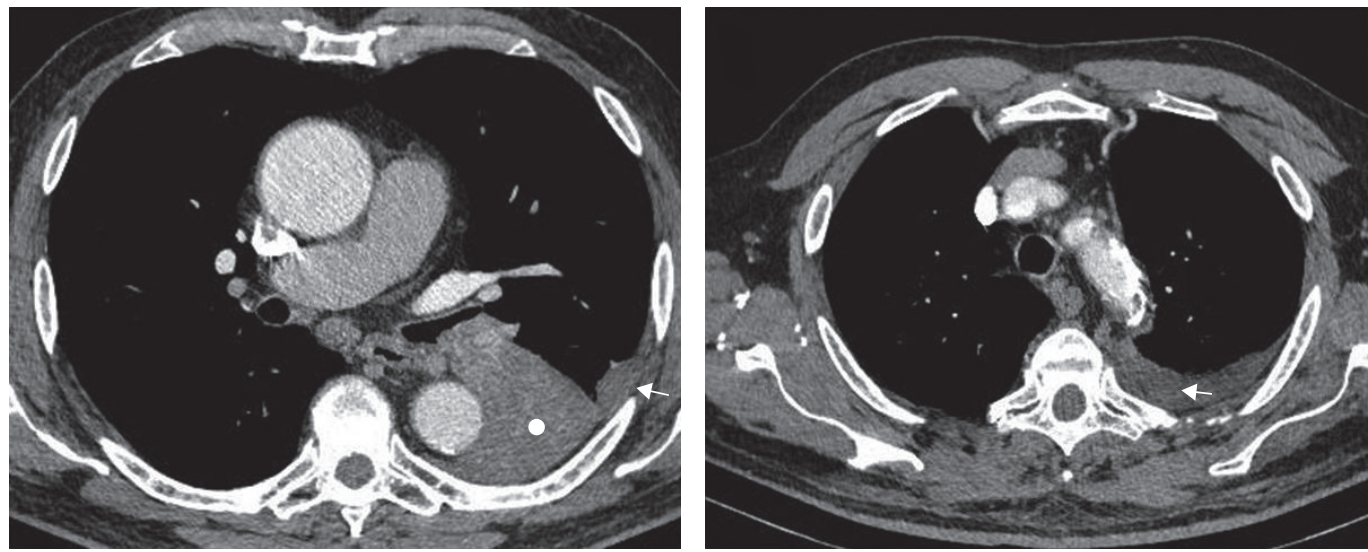

Figure 4 CT images with the notable features highlighted. The dot indicates collapse of the left lower lobe and the arrows indicate minimal left pleural effusion.

\section{Answer 2}

The CT scan of the thorax (figure 4) shows an obstruction of the proximal left lower lobe bronchus with complete collapse of the left lower lobe. There is also a mild left pleural effusion, and some mediastinal lymph nodes.

A malignant obstruction should be suspected. The most appropriate next step is to perform a bronchoscopy to obtain tissue for histopathological diagnosis.

Bronchoscopy showed a round mass totally obstructing the left lower lobe bronchus (figure 5). Biopsy of the mass revealed a "benign inflammatory polyp". Bronchial aspiration was negative for bacteriology and acid-fast bacilli on direct examination.

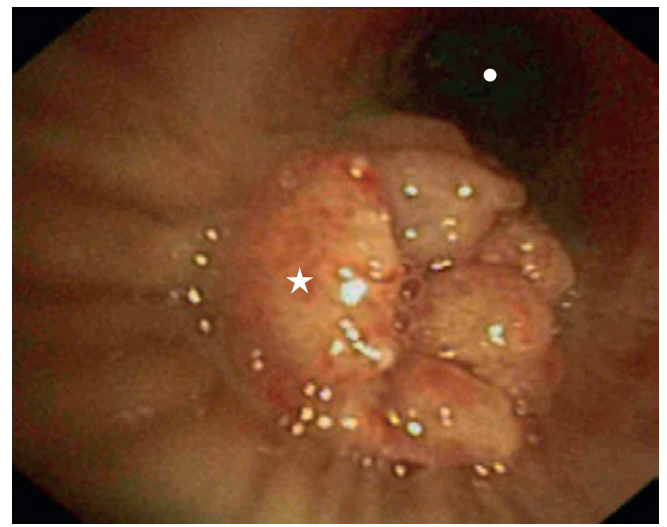

Figure 5 Bronchoscopy. The star indicates a round mass totally obstructing the left lower lobe bronchus and the dot indicates the left upper lobe bronchus.

\section{Task 3}

How would you manage this patient?

\section{Answer 3}

The final diagnosis is an obstructive pneumonia in a collapsed lower lobe, because of complete obstruction of the left lower lobe bronchus by a tumour that is probably benign.

Empiric antibiotics should be started as soon as possible. In this case, the patient was treated with ceftriaxon 2 g.day ${ }^{-1}$ and levofloxacin

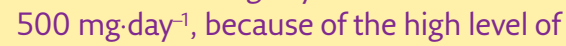
bacterial resistance in Vietnam, with clinical improvement (apyrexia and reduced cough) and regression of the inflammatory markers.

A lobectomy or bronchoscopic clearance of the left lower lobe bronchus should be discussed after evaluation of the extent of the tumour and cardiopulmonary function. The two main reasons for this are as follows.

1) Airway clearance is the only way to totally cure the obstructing pneumonia. Without clearance, there is a high risk of new infection with more and more resistant bacteria.

2) The pathology result is not convincing. $A$ new biopsy should be performed with more material to reach a more precise diagnosis and to definitively rule out lung cancer.

After 2 weeks of medical treatment, the chest radiograph showed persistence of total left lower lobe collapse despite the good evolution of infection. Positron emission tomography showed increased uptake by the tumour obstructing the left lower lobe (maximum standardised uptake value of 7), but no other lesions. Brain magnetic resonance imaging was normal. Spirometry showed a moderate mixed non-reversible pattern (forced expired volume in $1 \mathrm{~s}$ (FEV1) of $1400 \mathrm{~mL}, 51 \%$ of normal predicted value; forced vital capacity (FVC) $2040 \mathrm{~mL}, 53 \%$ of normal predicted value; and FEV1/FVC 68\%). A ventilation/perfusion scan with 


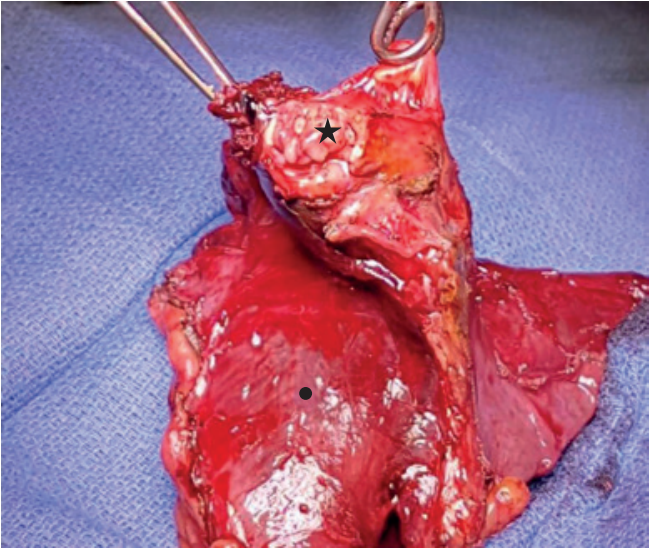

Figure 6 Left lower lobectomy. The star indicates the tumour and the dot the collapsed left lower lobe.

quantification showed a decreased perfusion in the left lower lobe, with a perfusion of $33 \%$ in the left lung and $67 \%$ in the right lung, and a ventilation of $31 \%$ in the left lung and $69 \%$ in the right lung. Echocardiography and cardiac exercise tests were normal.

The patient underwent a left lower lobectomy in France (figure 6), 1 month after presentation. The post-operative period was uneventful. Pathology concluded a proximal pedunculated in the left lower bronchus hamartoma constituted with fat and chondromyxoid material, $4 \times 1.4 \mathrm{~cm}$ in size. The left lower lobe was totally collapsed with chronic organising pneumonia, bronchiectasis and inflammatory pleural adhesion.

In a follow-up consultation in Vietnam 1 month later, the patient was asymptomatic, in good general status with only post-operative changes on the chest radiograph and CT scan of the thorax.

\section{Discussion}

Hamartoma is the most common benign neoplasm involving the lung. It usually presents as a peripheral mass and rarely as an endobronchial lesion [1]. Endobronchial hamartoma is usually pedunculated and originates from the proximal portion of the bronchial tree, without any predominant location [1]. It contains components of mature cartilage, muscle, fat, fibrous tissue and epithelium. In contrast to intrapulmonary lesions, endobronchial hamartoma is most often symptomatic with recurrent respiratory infections, haemoptysis, dyspnoea or wheezing [1-3].

Radiological findings show atelectasis, pleural effusion, bronchiectasis and parenchymatous consolidation as result of chronic bronchial obstruction. Sometimes, a hilar enlargement can be observed [1-3]. A CT scan of the thorax shows the same post-obstructive process and an endobronchial heterogeneous fatty, sometimes calcified, lesion without contrast enhancement [1-3]. Multiplanar reformation images may be helpful to detect intratumoural fat and calcification, and the relationship between the tumour and the bronchi [4]. On bronchoscopy it appears as an exophytic or polypoidal mass with a smooth well limited surface and without signs of submucosal infiltration [5].

For endobronchial hamartomas, a large resection such as lobectomy is preferred to endoscopic treatment with rigid bronchoscopy or laser when there is an irreversibly damaged lung after the obstruction. In other cases, bronchoscopic removal is very efficient $[1-3,5]$.

A few cases of recurrent or multiple hamartomas or of locoregional extension have been reported; however, most of the time, endobronchial hamartoma is a benign lesion without any recurrence or malignant evolution.

\section{Conflict of interest}

None declared.

\section{References}

1. Jhun BW, Lee $\mathrm{KJ}$, Jeon $\mathrm{K}$, et al. The clinical, radiological, and bronchoscopic findings and outcomes in patients with benign tracheobronchial tumors. Yonsei Med J 2014; 55: 84-91.

2. Cosio BG, Villena V, Echave-Sustaeta J, et al. Endobronchial Hamartoma. Chest 2002; 122: 202-205.

3. Prohm P, Winter J, Schmucker P. Pulmonary hamartoma. Thorac Cardiovasc Surg 1982; 30: 302-305.
4. Xiang Z, Zhu Al, Zhong G, et al. Diagnostic value of using multiplanar reformation images. Case report for rare endotracheal hamartomas. Medicine 2017; 96: e8231.

5. Kim SA, Um SW, Song JU, et al. Bronchoscopic features and bronchoscopic intervention for endobronchial hamartoma. Respirology 2010; 15: 150-154. 